\title{
The role of anisotropy and inhomogeneity in Lemaitre-Tolman-Bondi collapse
}

\author{
Filipe C. Mena ${ }^{1,3}$, Brien C. Nolan ${ }^{2}$ \& Reza Tavakol ${ }^{3}$ \\ ${ }^{1}$ Departamento de Matemática, Universidade do Minho, Campus de Gualtar, 4710 Braga, Portugal \\ 2 School of Mathematical Sciences, Dublin City University, Glasnevin, Dublin 9, Ireland \\ 3 Astronomy Unit, School of Mathematical Sciences, Queen Mary, University of London, London E1 4NS, U.K.
}

(November, 2003)

\begin{abstract}
We study the effects of shear and density inhomogeneities in the formation of naked singularities in spherically symmetric dust space-times. We find that in general neither of these physical features alone uniquely specifies the end state of the gravitational collapse. We do this by (i) showing that, for open sets of initial data, the same initial shear (or initial density contrast) can give rise to both naked and covered solutions. In particular this can happen for zero initial shear or zero initial density contrast; (ii) demonstrating that both shear and density contrast are invariant under a one parameter set of linear transformations acting on the initial data set and (iii) showing that asymptotically (near the singularities) one cannot in general establish a direct relationship between the rate of change of shear (or density contrast) and the nature of the singularities. However, one can uniquely determine the nature of the singularity if both the initial shear and initial density contrast are known.

These results are important in understanding the effects of the initial physical state and in particular the role of shear, in determiming the end state of the gravitational collapse.
\end{abstract}

PACS: 04.20.Dw, 04.20.Ex

Keywords: Cosmic censorship, naked singularity, black hole

\section{INTRODUCTION}

An important outcome of general relativity has been to show that subject to a number of physically reasonable assumptions, the final state of gravitational collapse will be singular in a range of settings (see e.g. [1]). An outstanding question concerns the nature of the resulting singularities and in particular whether and under what conditions they may be naked or black holes [2,3]. Over the recent years a great deal of effort has gone into the study of these questions. These have mainly concentrated on collapse in spherically symmetric settings and involve the study of collapse of scalar field $[4,5]$ as well as other matter sources including dust $[6,7]$, perfect fluids $[8,9]$, imperfect fluids [10-13] and null strange quark fluids [14,15].

All these studies show that the end state of the spherical collapse can be either a black hole or a naked singularity, depending upon the nature of the initial data and the kinematical properties near the singularity. Apart from the mathematical questions concerning the likelihood of each end-state, an important question from a physical point of view is how the nature of the end-state of collapse in the spherical settings may depend on physical characteristics of the regime under consideration. In particular, we ask how does the inhomogeneity and anisotropy influence the process and the final outcome of collapse?

This issue has been studied by a number of authors. Penrose [16] discusses the possibility that anisotropy might play a role in the occurence of naked singularities. Herrera et al. [17] considered a spherically symmetric collapsing space-time with an anisotropic fluid (with radial and tangential pressures) and showed that high density contrasts increase the radial velocity of collapse. They also proved that the local anisotropy of the pressure might have the same effect suggesting that this might lead to naked singularities (depending on some inequalities between radial and tangential pressures).

More recently, Joshi, Dadhich and Maartens [18] have also considered this question in the special case of marginally bounded Lemaitre-Tolman-Bondi (LTB) collapse and found that the shear associated with the initial distribution of matter fully determines the final outcome of collapse in these settings. In particular, they have found that the stronger the shear near the singularity the more likely it is to have a naked singularity. Soon after, Chan et al. [19] found a self-similar solution with heat-flow which was shear-free and, nevertheless, exhibited naked singularities.

Here, motivated by these previous results, we make a detailed study of the relation between the nature of the end-state of LTB collapse with anisotropy and inhomogeneity, by considering more general settings. We study how the choices of shear and density contrast functions can influence the nature of the gravitational singularities. In particular, we consider the behaviour of the shear function initially, asymptotically (near the singularity) as well as at intermediate times and study the effects of each on determining the end state of gravitational collapse. 
The structure of the paper is as follows: In section 2 we give a brief description of the spherical dust models. In Sections 3 and 4 we consider the effects of the shear on the end state of collapse. In section 5 we consider in turn the density contrast. Section 6 contains some remarks about space-time extensions after shell-crossing and initial data sets. Finally, we conclude in Section 7.

\section{SPHERICALLY SYMMETRIC DUST SPACE-TIMES}

The inhomogeneous spherically symmetric dust space-time can be represented by the Lemaitre-Tolman-Bondi (LTB) line element [20-22]

$$
d s^{2}=-d t^{2}+\frac{R^{\prime 2}}{1+E} d r^{2}+R^{2}\left(d \theta^{2}+\sin ^{2} \theta d \phi^{2}\right)
$$

where $r, \theta, \phi$ are comoving coordinates. The dot and prime denote differentiation with respect to $t$ and $r$ respectively and $R=R(r, t)$ and $E=E(r)$ are $C^{2}$ real functions such that $R(r, t) \geq 0$ and $E(r)>-1$. The matter-density is given by

$$
\rho(t, r)=\frac{m^{\prime}}{R^{2} R^{\prime}}
$$

where $m=m(r)$ is another $C^{2}$ real function such that $m(r)>0$. The evolution equation for the case of $\dot{R}<0$ (corresponding to gravitational collapse) takes the form

$$
\dot{R}=-\sqrt{\frac{m}{R}+E},
$$

and can be solved for different values of $E$ in the following parametric forms:

For $E<0$ :

$$
\begin{gathered}
R=\frac{m}{2(-E)}(1-\cos \eta) \\
(\eta-\sin \eta)=\frac{2(-E)^{\frac{3}{2}}}{m}\left(t_{c}-t\right)
\end{gathered}
$$

where $0<\eta<2 \pi$ and $t_{c}=t_{c}(r)$ is a third $C^{2}$ real function that corresponds to the time of arrival of each shell $r$ to the central singularity. Note that here and below, $t=t_{c}(r)$ corresponds to $\eta=0$.

For $E>0$ :

$$
\begin{gathered}
R=\frac{m}{2 E}(\cosh \eta-1), \\
(\sinh \eta-\eta)=\frac{2 E^{\frac{3}{2}}}{m}\left(t_{c}-t\right)
\end{gathered}
$$

where $\eta>0$.

For $E=0$ :

$$
R=\left(\frac{9 m}{4}\right)^{\frac{1}{3}}\left(t_{c}-t\right)^{\frac{2}{3}}
$$

In what follows we find it also useful to work with the compact form for the solution to equation (3);

$$
t_{c}-t=\sqrt{\frac{R^{3}}{m}} G\left(-\frac{E R}{m}\right)
$$

where $G$ is a positive real function given by 


$$
\begin{array}{lll}
G(x)=\frac{\arcsin (\sqrt{x})}{x^{3 / 2}}-\frac{\sqrt{1-x}}{x}, & \text { for } & 1 \geq x>0 \\
G(x)=\frac{2}{3}, & \text { for } \quad x=0 \\
G(x)=\frac{-\operatorname{arcsinh}(\sqrt{-x})}{(-x)^{3 / 2}}-\frac{\sqrt{1-x}}{x}, \text { for } \quad 0>x>-\infty .
\end{array}
$$

Using the coordinate freedom to rescale

$$
R(0, r)=r
$$

at an initial time $t=0$, equation $(7)$ gives

$$
t_{c}(r)=\frac{r^{3 / 2} G(p)}{\sqrt{m}}
$$

where $p=-r E / m$. So, the initial data set is given by $\mathcal{I}=\{m(r), E(r)\}$.

We note that the metric (1) can be matched at a boundary, say $r=r_{d}=$ const., to the Schwarzschild metric in the exterior region. Thus the scenario here is that of a collapsing compact matter region matched to an exterior Schwarzschild space-time.

We shall refer to a singularity as naked if there is a family of future directed non-spacelike geodesics which terminate at the singularity in the past. In spherical symmetry, if there are no radial-null geodesics emerging from a central singularity then the singularity is necessarily censored [23]. Therefore we shall only be concerned with outgoing radial null geodesics which, as can be seen from (1), correspond to the solution of the differential equation

$$
\frac{d t}{d r}=\frac{R^{\prime}}{\sqrt{1+E}}
$$

One can rewrite this equation as (see [6])

$$
\frac{d R}{d u}=\frac{1}{u^{\prime}}\left(R^{\prime}+\dot{R} \frac{d t}{d r}\right)=\left(1-\sqrt{\frac{E+\Lambda / X}{1+E}}\right) H(X, u),
$$

where

$$
\begin{aligned}
H(X, u) & =\left(\eta_{u}-\beta_{u}\right) X+ \\
& \left(\Theta_{u}-\left(\eta_{u}-\frac{3}{2} \beta_{u}\right) X^{\frac{3}{2}} G(-P X)\right) \sqrt{P+\frac{1}{X}}
\end{aligned}
$$

and

$$
\begin{aligned}
& X=\frac{R}{u}, \eta=r \frac{m^{\prime}}{m}, \eta_{u}=\eta \frac{u}{r u^{\prime}}, \beta=r \frac{E^{\prime}}{E}, \beta_{u}=\beta \frac{u}{r u^{\prime}}, \\
& P=\frac{u E}{m}, p=-r \frac{E}{m}, \Theta_{u}=\Theta \frac{\sqrt{r}}{\sqrt{u} u^{\prime}}, \Lambda=\frac{m}{u}, \\
& \Theta=\frac{\sqrt{m}}{\sqrt{r}} t_{c}^{\prime}(r)=\frac{1+\beta-\eta}{\sqrt{1-p}}+\left(\eta-\frac{3}{2} \beta\right) G(p),
\end{aligned}
$$

with the positive real function $u=u(r)$ being monotonically increasing and such that $u(0)=0$. Later on we will specify $u(r)=r^{3}$. In the cases where $E(r)=0$ we will take $\beta(r)=0$. Following [6], we use the algebraic equation in $X_{0}$ (obtained from (12))

$$
\left(1-\sqrt{\frac{E_{0}+\Lambda_{0} / X_{0}}{1+E_{0}}}\right) H\left(X_{0}, 0\right)-X_{0}=0,
$$

where the subscript ' 0 ' denotes the limit of the associated functions as $r \rightarrow 0$ (respectively $u \rightarrow 0$ ) along the geodesic, in order to demonstrate the existence of radial-null geodesics emanating from the singularity in spherical symmetric dust collapse.

An important outcome of past studies (e.g. [6]) is that the occurrence of black holes or naked singularities as final outcomes of collapse depend on the choice of initial data $\{m(r), E(r)\}$. This initial data set has to satisfy certain regularity conditions namely: 
1. there are no trapped surfaces initially, i.e. $R(0, r)>m(r)$, for $r>0$.

2. there is no shell-crossing during collapse, i.e. $R^{\prime}(t, r) \neq 0$, for $r>0$.

3. the initial matter-density $\rho(0, r)$ is non-zero at the center $r=0$.

\section{DEPENDENCE OF THE NATURE OF SINGULARITY ON SHEAR $\sigma(T, R)$}

In this section we study the shear $\sigma(t, r)$, in particular its behaviour in the approach to the singularity as well as the effects of changing $\sigma(t, r)$ on the nature of the singularity.

For the LTB metric the shear function (relative to the fluid congruence given by the 4 -velocity vector field) is given by

$$
\sigma(t, r)=\frac{\sqrt{3}}{3}\left(\frac{\dot{R}}{R}-\frac{\dot{R}^{\prime}}{R^{\prime}}\right)
$$

and its time derivative by

$$
\dot{\sigma}(t, r)=\frac{\sqrt{3}}{3}\left[\frac{\ddot{R}}{R}-\left(\frac{\dot{R}}{R}\right)^{2}-\frac{\ddot{R}^{\prime}}{R^{\prime}}+\left(\frac{\dot{R}^{\prime}}{R^{\prime}}\right)^{2}\right] .
$$

We start by recalling the following well-known result (see e.g. [7]):

Result 1 Consider a dust spherically symmetric collapsing space-time satisfying the regularity conditions. If $\sigma(t, r)=$ 0 then the final state of collapse is a covered singularity.

To proceed, we shall use the parametric solutions of the evolution equation to calculate $\dot{R} / R$ and $\dot{R}^{\prime} / R^{\prime}$ in cases $E<0$ and $E>0$. The case $E=0$ has already been considered in [18].

\section{A. CASE $E<0$}

In this case we have

$$
\dot{R}=\sqrt{-E} \frac{\sin \eta}{\cos \eta-1}
$$

together with

$$
R^{\prime}=\left(\frac{m}{2(-E)}\right)^{\prime}(1-\cos \eta)+\frac{m}{2(-E)} \sin \eta \frac{\partial \eta}{\partial r}
$$

and

$$
\dot{R}^{\prime}=(\sqrt{-E})^{\prime} \frac{\sin \eta}{\cos \eta-1}-\sqrt{-E} \frac{1}{\cos \eta-1} \frac{\partial \eta}{\partial r}
$$

where $\frac{\partial \eta}{\partial r}$ is given in the Appendix. We can then write

$$
\sigma(t, r)=\frac{\sqrt{3}}{3}\left(\frac{2(-E)^{3 / 2}}{m}\right)\left(\frac{-\sin \eta}{(\cos \eta-1)^{2}}+\frac{\frac{E^{\prime}}{2 E} \sin \eta-\frac{\partial \eta}{\partial r}}{\left(\frac{E^{\prime}}{E}-\frac{m^{\prime}}{m}\right)(\cos \eta-1)^{2}+\sin \eta(\cos \eta-1) \frac{\partial \eta}{\partial r}}\right) .
$$

It can be seen that $\sigma(t, r)$ is invariant under the transformation

$$
\begin{aligned}
\Phi_{a}: \mathcal{I} & \rightarrow \mathcal{I} \\
(m, E) & \mapsto\left(a^{3 / 2} m, a E\right),
\end{aligned}
$$


for an arbirtrary free parameter $a>0$. Note that $t_{c}(r)$ does change under this transformation, and so it is a nontrivial mapping on $\mathcal{I}$, i.e. it leads to a different LTB space-time. So, the same shear function $\sigma(t, r)$ can correspond to different initial data functions $m$ and $E$.

Since we are mainly interested in the behaviour of $\sigma(t, r)$ around the singularity we use an expansion around $\eta=0$ to obtain

$$
\sigma(t, r)=-\frac{2 \sqrt{3}(-E)^{3 / 2}}{3 m}\left(\frac{6}{\eta^{3}}+\frac{1}{2 \eta}+\frac{-2(A B)^{\prime}+\frac{2}{3} A B^{\prime}-B A^{\prime}}{A B^{2} t_{c}^{\prime}}-\frac{7}{120} \eta\right)+O\left(\eta^{2}\right)
$$

where $A=\frac{m}{2(-E)}$ and $B=\frac{2(-E)^{\frac{3}{2}}}{m}$.

\section{B. CASE $E>0$}

In this case, we have

$$
\dot{R}=\sqrt{E} \frac{\sinh \eta}{1-\cosh \eta}
$$

together with

$$
R^{\prime}=\left(\frac{m}{2 E}\right)^{\prime}(\cosh \eta-1)+\frac{m}{2 E} \sinh \eta \frac{\partial \eta}{\partial r}
$$

and

$$
\dot{R}^{\prime}=(\sqrt{E})^{\prime} \frac{\sinh \eta}{1-\cosh \eta}-\sqrt{E} \frac{1}{1-\cosh \eta} \frac{\partial \eta}{\partial r}
$$

where $\frac{\partial \eta}{\partial r}$ is given in the Appendix. We can then write

$$
\sigma(\eta, r)=\frac{\sqrt{3}}{3}\left(\frac{2 E^{3 / 2}}{m}\right)\left(\frac{-\sinh \eta}{(\cosh \eta-1)^{2}}+\frac{\frac{E^{\prime}}{2 E} \sinh \eta-\frac{\partial \eta}{\partial r}}{\left(\frac{E^{\prime}}{E}-\frac{m^{\prime}}{m}\right)(1-\cosh \eta)^{2}+\sinh \eta(1-\cosh \eta) \frac{\partial \eta}{\partial r}}\right) .
$$

We note that again $\sigma(t, r)$ is invariant under the transformation $\Phi_{a}(m, E)=\left(a^{3 / 2} m, a E\right)$, for an arbitrary free parameter $a>0$. As a result, the same shear function $\sigma(t, r)$ can correspond to different initial data functions $m$ and $E$. We use the above expressions and their expansions around $\eta=0$ to get

$$
\sigma(t, r)=-\frac{2 \sqrt{3} E^{\frac{3}{2}}}{3 m}\left(\frac{6}{\eta^{3}}-\frac{1}{2 \eta}+\frac{-2(A B)^{\prime}-B A^{\prime}}{A B^{2} t_{c}^{\prime}}+\frac{7 \eta}{120}\right)+O\left(\eta^{2}\right)
$$

where $A=\frac{m}{2 E}$ and $B=\frac{2 E^{\frac{3}{2}}}{m}$.

\section{Discussion}

The above results show that the shear $\sigma(t, r)$ for both $E>0$ and $E<0$ cases is invariant under the transformation $\Phi_{a}(m, E)=\left(a^{3 / 2} m, a E\right)$, with $a>0$. This is not true for $\Theta_{u}$. The fact that $\Theta_{u}$ is not invariant under $\Phi_{a}$ has the important consequence of allowing the same shear function to produce both naked singularities and black holes. As a simple example we take $m(r)=m_{3} r^{3}+m_{6} r^{6}$ and $E(r)=E_{2} r^{2}+E_{5} r^{5}$. In this case, letting $u=r^{3}$ we obtain from (15) the quartic equation

$$
2 X_{0}^{4}+\sqrt{m_{3}} X_{0}^{3}-3 \Theta_{u} X_{0}+3 \sqrt{m_{3}} \Theta_{u}=0
$$

with 


$$
\Theta_{u_{0}}=\frac{E_{5} / E_{2}-m_{6} / m_{3}}{\sqrt{1-p_{0}}}+\left(\frac{m_{6}}{m_{3}}-\frac{3}{2} \frac{E_{5}}{E_{2}}\right) G\left(p_{0}\right) .
$$

Taking $m_{3}=0.1, m_{6}=-0.9, E_{2}=-0.05, E_{5}=1$ we obtain positive roots for (30) which correspond to naked singularities. Applying $\Phi_{a}$ with $a=0.61$ to the previous initial data, we get black hole solutions instead and yet the same shear function $\sigma(t, r)$. Thus shear $\sigma(t, r)$ does not uniquely determine the final outcome of collapse.

In the approach to the singularity, the shear in both $E>0$ and $E<0$ cases can be approximated by

$$
\sigma(t, r)=-\frac{\sqrt{3}}{3} \frac{2|E|^{\frac{3}{2}}}{m}\left(\frac{6}{\eta^{3}}\right)+O\left(\eta^{-1}\right)
$$

This demonstrates that the behaviour of $\sigma(t, r)$ near the singularity for $E \neq 0$ depends in general on both $m$ and $E$, whereas in the $E=0$ case it depends only on $m$.

We can now study the shear strenght in the approach to the singularity depending upon the values of $|E|^{\frac{3}{2}} / m$ as well as the relationship between $|E|^{\frac{3}{2}} / m$ and the time of horizon formation $t_{H}$. The surface $t=t_{H}(r)$ is defined by $R=m$. Using (7), this gives

$$
t_{H}=t_{c}-m G(-E)
$$

Using the expression (10) for $t_{c}$, we have

$$
\frac{\partial t_{H}}{\partial m}=-\frac{1}{2} \frac{r^{3}}{m^{3 / 2}}\left(G(p)+2 p G^{\prime}(p)\right)-G(-E),
$$

where $p=-r E / m$. Now $G$ is positive, and it turns out that $G(p)+2 p G^{\prime}(p)$ is also positive, and so

$$
\frac{\partial t_{H}}{\partial m}<0
$$

Thus for fixed $E$, regardless of sign, $t_{H}$ does increase with decreasing $m$.

It is known that delays in the time of horizon formation can be associated with the increased likelihood of finding naked solutions. From (35) we find that smaller $m$ produces delays in $t_{H}$. Since smaller $m$ also induces stronger shear around the singularity (see equation (32)) one may be tempted to relate stronger shears with delays in $t_{H}$ and consequently with increased likelihood of finding naked solutions, as was done in [18] for $E=0$. However, for $E \neq 0$, this is not necessarily true since in general one needs both $m$ and $E$ to decide whether a solution is naked or not.

Now recall that for LTB, given an initial function $m$ one can always find a function $E$ such that a naked singularity solution exists $[6,24]$. So we may ask whether smaller $m$ values make it easier to find $E$ functions which give rise to naked singularities. In order to answer this question we also note that the part of the singularity surface $t_{c}(r)$ which can produce naked singularities is $t_{c}(0)$, so we are mainly interested in the behaviour of $\sigma(t, r)$ around $r=0$.

We take $C^{\infty}$ functions $m$ and $E$ and integers $N>2, M>3$ so that we can always write $m(r)=\sum_{i=3}^{N} m_{i} r^{i}$, with $m_{3} \neq 0$ and $E(r)=\sum_{j=2}^{M} E_{j} r^{j}$, with $E_{2} \neq 0$. In this case, we get around $r=0$

$$
\sigma(t, r)=-\frac{\sqrt{3}}{3} \frac{2\left|E_{2}\right|^{\frac{3}{2}}}{m_{3}}\left(\frac{6}{\eta^{3}}\right)+O\left(\eta^{-1}\right)
$$

and we find naked singularity solutions from equation (15) if and only if (see e.g. [6,7])

$$
\left.\Theta_{u_{0}} \in \mathbf{I}=\right] 0, m_{3}^{\frac{3}{2}}\left(\frac{13}{3}-\frac{5 \sqrt{3}}{2}\right)[\cup] m_{3}^{\frac{3}{2}}\left(\frac{13}{3}+\frac{5 \sqrt{3}}{2}\right),+\infty[.
$$

Now a measure for the interval $\mathbf{R}^{+} \backslash \mathbf{I}$ is

$$
5 \sqrt{3} m_{3}^{\frac{3}{2}} .
$$

It is then clear that the smaller $m_{3}$ the smaller will be the measure of $\mathbf{R}^{+} \backslash \mathbf{I}$ and consequently, there might exist examples where it is easier to find functions $E$ so that $\Theta_{u_{0}} \in \mathbf{I}$. In fact this is the case for a number of known examples (see e.g. [7] for a graphic illustration). However, this phenomena is not general. The problem arises from the fact that $\Theta_{u_{0}}$ depends not only on $m_{3}$ and $E_{2}$ but also on the higher order coeficients $m_{i}$ and $E_{j}$ which although do not appear in the asymptotic $(r \rightarrow 0)$ expression for $\sigma(t, r)$, can nevertheless be crucial to determine whether $\Theta_{u_{0}} \in \mathbf{I}$. 
This then tells us that the information in the vicinity of the singularity given by $E_{2}, m_{3}$, which determines the shear rate in (36), is not enough to determine the nature of the singularity. It is therefore crucial to be sufficiently far from the singular region in order to pick up information about higher order "inhomogeneous" coeficients which characterise the initial data.

We note that Joshi et. al. [18] have calculated $\sigma(t, r)$ and $\sigma(0, r)$ for the case $E=0$ and concluded that in approach to the singularity these two quantities change with the same rate in $r$. For the case $E \neq 0$, however, one cannot obtain expressions for $\sigma(t, r)$ which explicitly depend on $r$ and $t$ so one cannot compare analytically the rates of change of $\sigma(t, r)$ and $\sigma(0, r)$ in the approach to the singularity (as was done for $E=0$ ). Nevertheless, we prove in the next section that the conclusions for $E=0$ do not necessarily carry over to the general $(E \neq 0)$ case.

We also note that although the function $R$ has the same asymptotic behaviour in the approach to the singularity for all $E$, the shear function $\sigma(t, r)$ does not, since the dependence of $\sigma(t, r)$ on the function $E$ persists all the way down to the singularity for the cases where $E \neq 0$. Furthermore, although $R$ "forgets" the $E$ dependence in the approach to the singularity, the geodesic equations do not and this is crucial in the study of the nature of the singularities.

To summarize, in this section we have proved that (i) $\sigma(t, r)$ is invariant under the linear transformations $\Phi_{a}$, (ii) $\sigma(t, r)$ diverges in the approach to the singularity at a rate depending on $|E|^{3 / 2} / m$ and (iii) $\sigma(t, r)$ does not uniquely specify the nature of the singularity. We have also compared our results for $E \neq 0$ to the ones obtained for $E=0$ by Joshi et al. [18].

\section{DEPENDENCE OF THE NATURE OF SINGULARITY ON INITIAL SHEAR $\sigma(0, R)$}

In this section, we study the initial shear $\sigma(0, r)$ as well as its possible influence on the nature of the singularities. For $t=0$, given $R(0, r)=r$, one can derive

$$
\sigma(0, r)=\frac{\sqrt{3}}{3}\left(E+\frac{m}{r}\right)^{-\frac{1}{2}}\left[\frac{E^{\prime}}{2}-\frac{E}{r}-\frac{3 m}{2 r^{2}}+\frac{m^{\prime}}{2 r}\right] .
$$

The initial radial velocity of collapse is $v_{i}=\dot{R}(0, r)$. So, one can write

$$
\sigma(0, r)=\frac{\sqrt{3}}{3}\left(\frac{v_{i}}{r}-v_{i}^{\prime}\right)
$$

which implies that $\sigma(0, r)$ increases with $v_{i}$. This confirms, for the case of dust, the results of [17] according to which local anisotropy increases the radial velocity of collapse. Now, for $\dot{R}(t, r) \neq 0$, we have that $\sigma(0, r)=0$ if and only if

$$
\frac{1}{2} E^{\prime}-\frac{E}{r}-\frac{3}{2} \frac{m}{r^{2}}+\frac{1}{2} \frac{m^{\prime}}{r}=0 .
$$

Result 2 Consider a dust spherically symmetric space-time $(M, g)$ with $\sigma(0, r)=0$. Then the following are equivalent:

1. $\dot{\sigma}(0, r)=0$.

2. $(M, g)$ is spatially homogeneous and isotropic.

3. $\sigma(t, r)=0$, for all $t>0$.

Proof: Using (17) for $\sigma(0, r)=0$ we obtain

$$
\dot{\sigma}(t, r)=\frac{\sqrt{3}}{3}\left(\frac{\ddot{R}}{R}-\frac{\ddot{R}^{\prime}}{R^{\prime}}\right)
$$

which gives

$$
\dot{\sigma}(0, r)=\frac{\sqrt{3}}{6}\left[\frac{m^{\prime}}{r^{2}}-\frac{3 m}{r^{3}}\right]
$$

Note that this does not depend on $E$. The condition $\dot{\sigma}(0, r)=0$ implies $m(r)=m_{3} r^{3}$, which in turn from $\sigma(0, r)=0$ implies $E(r)=E_{2} r^{2}$. These correspond to spatially homogeneous initial data (see e.g. [7]). On the other hand, spatial homogeneity implies $\dot{\sigma}(t, r)=0 \bullet$ 
Result 2 indicates that, as expected in general, even if we start with zero initial shear in an inhomogeneous space-time, shear will be generated after evolution starts. This can also be seen from the relationship between $\dot{\sigma}(0, r)$ and $\rho(0, r)$ :

$$
\dot{\sigma}(0, r)=\frac{\sqrt{3}}{6}\left[\rho(0, r)-\frac{3 m}{r^{3}}\right] .
$$

This, however, is not the case for $E=0$ :

Result 3 Consider a dust spherically symmetric space-time with $E=0$. If $\sigma(0, r)=0$ then $\sigma(t, r)=0$.

Proof: For $E=0$, if $\sigma(0, r)=0$ then one must have $m=m_{3} r^{3}$ which gives $\dot{\sigma}(0, r)=0$. Now apply Result (2)

For the case of $E=0$, since shear depends only on $m$ there is a unique correspondence between $m$ and $\sigma(0, r)$ and one can therefore substitute the initial free function $m$ by $\sigma(0, r)$. One can then study how the initial shear influences the final outcome of collapse, as in [18]. In particular, if $\sigma(0, r)=0$ the final outcome of collapse is necessarily a black hole. An interesting question is whether this result necessarily holds for $E \neq 0$.

The situation is in fact more complicated in the case $E \neq 0$, where as we shall show the same initial shear can give rise to different outcomes. Before doing so we note that one can fine tune the initial data so that $\sigma(0, r)=0$ and $\dot{\sigma}(0, r) \neq 0$.

Result 4 Consider a dust spherically symmetric collapsing space-time with $m(r)=r^{3} g(r)$ and $E(r)=r^{2} Q(r)$, such that $g$ and $Q$ are $C^{2}$ real functions in $\left[0, r_{d}\right]$ with $g(0) \neq 0$ and $Q(0) \neq 0$. Then $\sigma(0, r)=0$ if and only if $Q^{\prime}+g^{\prime}=0$.

Proof: Follows directly from expression (38) •

Therefore there can exist situations where the initial shear is zero and nevertheless a naked singularity will be formed. Consider, for example, an initial data set $\mathcal{I}$ given by the functions $m(r)=m_{3} r^{3}+m_{6} r^{6}$ and $E(r)=E_{2} r^{2}+E_{5} r^{5}$. Taking the initial coefficients to be $E_{2}=m_{3}=0.1$ and $E_{5}=-m_{6}=1$ we ensure that $\sigma(0, r)=0$ and $\dot{\sigma}(0, r) \neq 0$ (this initial data also satisfies the regularity conditions). Solving (30) for these values one finds a positive root $X_{0}$ which therefore corresponds to a naked singularity.

Taking the same initial data except for the value of $m_{3}=0.2$ we find a black hole, while we still have $\sigma(0, r)=0$ and $\dot{\sigma}(0, r) \neq 0$. One can also find examples for $E_{2}<0$ such as $E_{2}=-0.05, m_{3}=0.1, E_{5}=-m_{6}=1$ giving a naked singularity and $E_{2}=-0.05, m_{3}=1, E_{5}=-m_{6}=1$ giving a black hole, both initial sets with $\sigma(0, r)=0$ and $\dot{\sigma}(0, r) \neq 0$.

Furthermore one can find open intervals in the coefficients of $m$ and $E$ such that the initial shear is zero and yet naked singularities form as in the next example: Taking $m(r)=m_{3} r^{3}+m_{6} r^{6}$ and $E(r)=E_{2} r^{2}+E_{5} r^{5}$ with $m_{6}+E_{5}=0$, then from $(31)$ we obtain

$$
\Theta_{u_{0}}=\frac{E_{5}}{E_{2}}\left(\sqrt{1-p_{0}}+\left(p_{0}-\frac{3}{2}\right) G\left(p_{0}\right)\right),
$$

with $p_{0}=-E_{2} / m_{3}$. Taking the elliptic case for which $0<p_{0} \leq 1$, i.e.

$$
E_{2} \in\left[-m_{3}, 0[\right.
$$

and consequently $2 / 3<G\left(p_{0}\right) \leq \pi / 2$. In this case $\sqrt{1-p_{0}}+\left(p_{0}-3 / 2\right) G\left(p_{0}\right)$ is always bounded and negative. So, in order to have $\Theta_{u_{0}}>0$ and therefore avoid shell-crossing we take $E_{5}>0$. Furthermore in order to ensure that $\Theta_{u_{0}}$ falls in one of the intervals given by $(37)$, e.g. $\Theta_{u_{0}}>m_{3}^{2 / 3}\left(\frac{13}{3}+\frac{5 \sqrt{3}}{2}\right)$, we impose

$$
\left.E_{5} \in\right] \frac{E_{2} m_{3}^{2 / 3}\left(\frac{13}{3}+\frac{5 \sqrt{3}}{2}\right)}{\sqrt{1-p_{0}}+\left(p_{0}-3 / 2\right) G\left(p_{0}\right)},+\infty[\text {. }
$$

So, $\sigma(0, r)=0$ and naked singularities as final state of collapse can be achieved for open subsets (in the sense made precise above) of the initial data. The possibility of having the same initial shear with different end states for the collapse can also be found for non-zero initial shear. This can be shown by generalising Result 4:

Result 5 Consider a dust spherically symmetric collapsing space-time with initial data as given in Result 4. Then two different sets of initial data $\left\{m^{(1)}, E^{(1)}\right\}$ and $\left\{m^{(2)}, E^{(2)}\right\}$ have the same initial shear function $\sigma(0, r)$ if and only if $h^{(1)^{\prime}}+Q^{(1)^{\prime}}=h^{(2)^{\prime}}+Q^{(2)^{\prime}}$. 
An example of this is the naked singularity solution obtained for $i=6$ and $j=5$ with $m_{3}=0.1, E_{2}=-0.05, m_{6}=$ $-0.9, E_{5}=1$, and the black hole solution $m_{3}=1, E_{2}=-0.95, m_{6}=-0.9, E_{5}=1$ both having the same non-zero initial shear profile. Again one can find open subsets of the initial data which have the same non-zero initial shear and yet different outcomes.

To summarize, in this section we have proved that (i) the same initial shear can result in two different outcomes black holes and naked singularities depending on $\{m, E\}$, so initial shear does not uniquely determine the final state of collapse; (ii) it is possible to have zero initial shear and a naked singularity endstate, and (iii) these results can be obtained for open sets of initial data.

\section{DEPENDENCE OF THE NATURE OF SINGULARITY ON INHOMOGENEITY}

The studies of the previous sections for the shear can now be repeated for inhomogeneity measures. In this section, we study the influence of the density contrast on the nature of the singular endstates. We shall use the pointwise density contrast measure

$$
\rho^{\prime}(t, r)=\frac{m^{\prime \prime}}{R^{\prime} R^{2}}-\frac{m^{\prime} R^{\prime \prime}}{R^{2}\left(R^{\prime}\right)^{2}}-\frac{2 m^{\prime}}{R^{3}}
$$

and start by recalling the following well-known result:

Result 6 Consider a dust spherically symmetric collapsing space-time satisfying the regularity conditions. If $\rho^{\prime}(t, r)=$ 0 then the final state of collapse is a black hole.

We shall therefore consider the cases where $\rho^{\prime}(t, r) \neq 0$. If we now use the formulae in the Appendix and substitute for $R, R^{\prime}$ and $R^{\prime \prime}$ in equation (47) we obtain, after expanding around $\eta=0$,

$$
\rho^{\prime}(t, r)=-\left(\frac{4|E|}{m}\right)^{3} \frac{m^{\prime}}{\eta^{6}}+O\left(\eta^{-3}\right) .
$$

Thus as in the case of shear, the inhomogeneity measure $\rho^{\prime}(t, r)$ depends asymptotically on both $E$ and $m$. In order to prove that $m^{\prime}(4|E| / m)^{3}$ does not uniquely determine the nature of the singularity we note that this quantity is invariant under the linear transformation $\Phi_{a}(m, E)=\left(a^{3 / 2} m, a E\right)$, for an arbitary free parameter $a>0$. In fact, by substituting $R, R^{\prime}$ and $R^{\prime \prime}$ in (47) we also find that the all time function $\rho^{\prime}(t, r)$ is itself invariant under $\Phi_{a}$. Interestingly $\Phi_{a}$ also leaves invariant the shear function $\sigma(t, r)$ and the examples of section III C can also be used here in order to show that the same function $\rho^{\prime}(t, r)$ can give rise to both naked singularities and black holes.

We now study the initial density contrast function $\rho^{\prime}(0, r)$. From $(47)$ we obtain for $t=0$ and $R(0, r)=r$

$$
\rho^{\prime}(0, r)=\frac{m^{\prime \prime}}{r^{2}}-\frac{2 m^{\prime}}{r^{3}}
$$

so $\rho^{\prime}(0, r)$ does not depend on $E$, which makes it clear that a given $\rho^{\prime}(0, r)$ can correspond to both black holes and naked singularities, depending on the choice of $E$ (see e.g [7]). So $\rho^{\prime}(0, r)$ cannot uniquely determine the final outcome of collapse, except in the $E=0$ case. An interesting question is whether there are naked singularity solutions for $\rho^{\prime}(0, r)=0$. In order to answer this question we start by recalling a simple result:

Result 7 Consider a dust spherically symmetric space-time with $\rho^{\prime}(0, r)=0$. Then the space-time is spatially homogeneous and isotropic if and only if $\sigma(0, r)=0$.

Proof: If $\rho^{\prime}(0, r)=0$ then $m=m_{3} r^{3}, m_{3} \in \mathbf{R} \backslash 0$, in which case $\sigma(0, r)=0$ if and only if $E=E_{2} r^{2} \bullet$

We recall that in the $E=0$ case the space-time is spatially homogeneous and isotropic if and only if $\rho^{\prime}(0, r)=0$. We shall then take the inhomogeneous $E \neq 0$ cases where it might be possible, for $\sigma(0, r) \neq 0$, to find initial data such that $\rho^{\prime}(0, r)=0$ and nevertheless have a naked solution. As an example of this we take $m(r)=m_{3} r^{3}, m_{3} \in \mathbf{R}^{+}$, and $E(r)=E_{2} r^{2}+E_{5} r^{5}$ such that

$$
\Theta_{u_{0}}=\frac{E_{5}}{E_{2}}\left(\frac{1}{\sqrt{1-p_{0}}}-\frac{3}{2} G\left(p_{0}\right)\right) .
$$

By taking the elliptic case with $E_{2} \in \mathbf{R}^{-}$and 


$$
\left.E_{5} \in\right] \frac{E_{2} m_{3}^{\frac{3}{2}}\left(\frac{13}{3}+\frac{5 \sqrt{3}}{2}\right)}{\frac{1}{\sqrt{1-p_{0}}}-\frac{3}{2} G\left(p_{0}\right)}, 0[
$$

we ensure both that $\Theta_{u_{0}}>0$ and that equation (15) has positive roots corresponding to naked singularities.

To summarize, in this section we have shown that (i) $\rho^{\prime}(t, r)$ is invariant under the linear transformations $\Phi_{a}$, (ii) $\rho^{\prime}(t, r)$ diverges in the approach to the singularity at a rate depending on $m^{\prime}(|E| / m)^{3}$, (iii) $\rho^{\prime}(t, r)$ does not uniquely specify the nature of the singularity, (iv) the same initial inhomogeneity function $\rho^{\prime}(0, r)$ can result in two different outcomes black holes and naked singularities depending on $\{m, E\}$, so $\rho^{\prime}(0, r)$ does not uniquely determine the final state of collapse and (v) it is possible to have zero initial density contrast and a naked singularity endstate. These results can be obtained for open sets of initial data.

\section{NON-UNIQUENESS, SHELL-CROSSING AND THE INITIAL DATA SET}

Finally, we consider two important issues in this section: (I) Non-uniqueness of space-time extensions after shellcrossing singularities and (II) Initial data sets based on initial shear and initial density contrast functions.

(I) The theme of our discussion has been how certain quantities which have been suspected of determining the outcome (naked singularity or black hole) of spherical dust collapse in fact fail to do so. We have emphasised the significance of the well-known fact that one needs to know both $E$ and $m$ in order to make this prediction. However there is another situation in which even the knowledge of both these functions is not enough to completely determine the space-time; that is when the initial data are such that shell-crossing singularities can occur. It has been shown recently how one can construct a dynamical extension - i.e. one based on the field equations - through a shell-crossing singularity and obtain global weak solutions of the field equations for $R>0, t>0$ [25]. However these weak solutions are not unique to the future of the shell-crossing. On the other hand, the earliest point of the shell-crossing is always globally naked, regardless of how the extension is constructed, so the non-uniqueness is not so severe as to allow the same initial data give rise to either a black hole or a naked singularity.

(II) As we have seen, neither $\sigma(0, r)$ nor $\rho^{\prime}(0, r)$ alone can uniquely determine the final outcome of collapse. However, both functions might form a perfectly good initial data set. The question is how from a set of observables $\mathcal{J}=\left\{\rho^{\prime}(0, r), \sigma(0, r)\right\}$, given on the onset of collapse, can one predict (uniquely) the final outcome of collapse.

Now $m(r)$ is uniquely determined by $\rho^{\prime}(0, r)$ through the linear ODE (49):

$$
m^{\prime \prime}-\frac{2}{r} m^{\prime}=r^{2} \rho^{\prime}(0, r),
$$

with initial conditions $m(0)=m^{\prime}(0)=0$. The shear $\sigma(0, r)$ can be written as a function of a new variable $w(r)=$ $E+m / r$ :

$$
\sigma(0, r)=\frac{\sqrt{3}}{3} w^{-\frac{1}{2}}\left(-\frac{w}{r}+\frac{1}{2} w^{\prime}\right),
$$

with initial conditions $w(0)=w^{\prime}(0)=0$. So, from $\sigma(0, r)$ one can determine $w(r)$ by solving the linear ODE

$$
y^{\prime}-\frac{2}{r} y=\sqrt{3} \sigma(0, r),
$$

where $w=y^{2}$. So, using (52), we can get $m(r)$ from $\rho^{\prime}(0, r)$. Knowing $m(r)$ and $\sigma(0, r)$ we can determine $E$ from (54). So, if we know both the density contrast $\rho^{\prime}(0, r)$ and the anisotropy measure $\sigma(0, r)$ we can recover both $E$ and $m$, and so predict the outcome of collapse. Since this process relies on solving linear ODEs, then one can ensure that solutions exist and are unique, for the given set of initial conditions.

It is worthwhile noting that even though the set $\left\{\sigma(0, r), \rho^{\prime}(0, r)\right\}$ can specify the initial data uniquely, the set $\left\{\sigma(t, r), \rho^{\prime}(t, r)\right\}$ specified at any other time cannot. The reason is that the latter is invariant under the one-parameter set of linear transformations $\Phi_{a}$ acting on the initial data set $\{m, E\}$, while the former is not and this is because even though initially one can use the rescaling to define $R(0, r)=r$, this cannot be repeated at subsequent times. 


\section{CONCLUSIONS}

We have considered LTB collapsing models in order to study the influence of inhomogeneity and anisotropy in the nature of the resulting singularities. We have studied the shear and density contrast in three ways: (i) by considering the all time functions $\sigma(t, r)$ and $\rho^{\prime}(t, r)$, (ii) by considering the asymptotic functions $\sigma(t, r)$ and $\rho^{\prime}(t, r)$ and (iii) by considering the initial functions $\sigma(0, r)$ and $\rho^{\prime}(0, r)$.

We have found that both shear $\sigma(t, r)$ and density contrast $\rho^{\prime}(t, r)$ are invariant under a one parameter set of linear transformations acting on the initial data set $\{m, E\}$. We have also found that asymptotically one cannot establish, in general, a direct link between the strenght of shear (or density constrast) and the nature of the singularities. Finally we have found that the same initial shear (or initial density contrast) can give rise to both naked singularities and black holes, depending on the choice of the initial density contrast (or initial shear, respectively). In particular this can happen for zero initial shear or zero initial density contrast. These results were obtained for open sets of initial data. We conclude that neither anisotropy nor inhomogeneity features as given by the shear or density contrast can alone uniquely specify the end state of collapse for $E \neq 0$, in contrast to the results established for $E=0$ in [18].

However, we have proved that if we know both the initial shear $\sigma(0, r)$ and the initial density contrast $\rho^{\prime}(0, r)$ functions then, given the appropriate initial conditions, we can determine uniquely the initial LTB data functions $\{m, E\}$ and hence determine the nature of singularity. In this sense one needs information about both the inhomogeneity and anisotropy at the initial hypersurface in order to predict the outcome of the collapse. This is important for understanding the effects of the initial physical state in determiming the end state of gravitational collapse.

\section{Acknowledgments}

FCM thanks Sérgio Gonçalves for interesting discussions, British Council/CRUP for grant N13/03, Fundação Calouste Gulbenkian for grant 21-58348-B and Centro de Matemática, Universidade do Minho, for support.

VIII. APPENDIX

$$
\begin{aligned}
& \text { A. } E<0 \\
& \frac{\partial \eta}{\partial t}=\frac{2(-E)^{3 / 2}}{m} \frac{1}{\cos \eta-1}<0 \\
& \frac{\partial \eta}{\partial r}=\frac{1}{1-\cos \eta}\left[\frac{m}{2(-E)^{\frac{3}{2}}}\left(\frac{2(-E)^{\frac{3}{2}}}{m}\right)^{\prime}(\eta-\sin \eta)+\frac{2(-E)^{\frac{2}{3}}}{m} t_{c}^{\prime}\right] \\
& R^{\prime \prime}=\left(\frac{m}{2(-E)}\right)^{\prime \prime}(1-\cos \eta)+2\left(\frac{m}{2(-E)}\right)^{\prime} \sin \eta \frac{\partial \eta}{\partial r}+\frac{m}{2(-E)}\left(\cos \eta \frac{\partial \eta}{\partial r}+\sin \eta \frac{\partial^{2} \eta}{\partial r^{2}}\right) \\
& \text { B. } E>0 \\
& \frac{\partial \eta}{\partial t}=-\frac{2 E^{\frac{3}{2}}}{m} \frac{1}{\cosh \eta-1}<0 \text {. } \\
& \frac{\partial \eta}{\partial r}=\frac{1}{\cosh \eta-1}\left[\frac{m}{2 E^{3 / 2}}\left(\frac{2 E^{\frac{3}{2}}}{m}\right)^{\prime}(\sinh \eta-\eta)+\frac{2 E^{\frac{3}{2}}}{m} t_{c}^{\prime}\right] \\
& R^{\prime \prime}=\left(\frac{m}{2 E}\right)^{\prime \prime}(\cosh \eta-1)+2\left(\frac{m}{2 E}\right)^{\prime} \sinh \eta \frac{\partial \eta}{\partial r}+\frac{m}{2 E}\left(\cosh \eta \frac{\partial \eta}{\partial r}+\sinh \eta \frac{\partial^{2} \eta}{\partial r^{2}}\right)
\end{aligned}
$$


[1] Hawking S W \& Ellis G F R, The large scale structure of space-time, (Cambridge, Cambridge University Press, 1985)

[2] Penrose R, Nuovo Cimento, 1 (1969) 252

[3] Wald R, published electronically as preprint gr-qc/9710068 (1997)

[4] Christodoulou D, Ann. of Math., 149 (1999) 183

[5] Martin-Garcia J M \& Gundlach C, Phys. Rev. D68 (2003) 024011

[6] Dwivedi I H \& Joshi P S, Class. Quant. Grav., 14 (1997) 1223

[7] Mena F C, Tavakol R \& Joshi P S, Phys. Rev. D62 (2000) 044001

[8] Giambo R, Giannoni F, Magli G \& Piccione P, preprint gr-qc/0303043 (2003)

[9] Harada T \& Maeda H, Phys. Rev. D63 (2001) 084022

[10] Harada T, Nakao K \& Iguchi H, Class. Quant. Grav. 16 (1999) 2785

[11] Giambo R, Giannoni F, Magli G \& Piccione P, Commun. Math. Phys. 235 (2003) 545

[12] Gonçalves S, Jhingan S \& Magli G, Phys. Rev. D65 (2002) 064011

[13] Joshi P S \& Goswani R, preprint gr-qc/0206042 (2002)

[14] Ghosh S G \& Dadhich N, Gen. Rel. Grav. 35 (2003) 359

[15] Harko T \& Cheng K S, Phys. Lett. A266 (2000) 249

[16] Penrose, R, in General relativity: An Einstein Centenary Survey, Eds. Hawking, S W \& Israel W, (Cambridge: Cambridge University Press, 1979)

[17] Herrera L, Di Prisco A, Hernández-Pastora J L \& Santos N O, Phys. Lett. A237 (1998) 113

[18] Joshi P S, Dadhich N \& Maartens R, Phys. Rev. D65 (2002) 101501

[19] Chan R, da Silva M F A \& Villas da Rocha J F, Int. J. Mod. Phys. D12 (2003) 347

[20] Lemaitre G, Ann. Soc. Sci. Bruxelles, A53 (1933) 51

[21] Tolman R C, Proc. Nat. Acad. Sci., 20 (1934) 169

[22] Bondi H, Mon. Not. R. Astron. Soc., 107 (1947) 410

[23] Nolan B C, Mena F C \& Gonçalves S, Phys. Lett. A294 (2002) 122

[24] Saraykar R V \& Ghate S H, Class. Quant. Grav. 16 (1999) 281

[25] Nolan B C, Class. Quant. Grav., 20 (2003) 575 